\title{
Sensorimotor alpha activity is modulated in response to the observation of pain in others
}

\author{
Stephen Whitmarsh ${ }^{1,2}{ }^{*}$, Ingrid L. C. Nieuwenhuis ${ }^{3}$, Henk P. Barendregt ${ }^{2}$ and Ole Jensen ${ }^{1}$ \\ ' Donders Institute for Brain, Cognition and Behaviour, Radboud University Nijmegen, Nijmegen, Netherlands \\ 2 Institute for Computing and Information Sciences, Radboud University Nijmegen, Nijmegen, Netherlands \\ 3 Sleep and Neuroimaging Laboratory, Department of Psychology, University of California Berkeley, Berkeley, CA, USA
}

\section{Edited by:}

Srikantan S. Nagarajan, University of

California San Francisco, USA

\section{Reviewed by:}

Juliana Yordanova, Bulgarian Academy of Sciences, Bulgaria

Douglas O. Cheyne, Hospital for Sick

Children, Canada

*Correspondence:

Stephen Whitmarsh, Donders Institute for Brain, Cognition and Behaviour,

Radboud University Nijmegen,

Kapitte/weg 29, 6525 EN Nijmegen, Netherlands.

e-mail:s.whitmarsh@cs.ru.nl
The perception-action account of empathy states that observation of another person's state automatically activates a similar state in the observer. It is still unclear in what way ongoing sensorimotor alpha oscillations are involved in this process. Although they have been repeatedly implicated in (biological) action observation and understanding communicative gestures, less is known about their role in vicarious pain observation. Their role is understood as providing a graded inhibition through functional inhibition, thereby streamlining information flow through the cortex. Although alpha oscillations have been shown to have at least visual and sensorimotor origins, only the latter are expected to be involved in the empathetic response. Here, we used magnetoencephalography, allowing us to spatially distinguish and localize oscillatory components using beamformer source reconstruction. Subjects observed realistic pictures of limbs in painful and no-pain (control) conditions. As predicted, time-frequency analysis indeed showed increased alpha suppression in the pain condition compared to the no-pain condition. Although both pain and no-pain conditions suppressed alpha- and beta-band activity at both posterior and central sensors, the pain condition suppressed alpha more only at central sensors. Source reconstruction localized these differences along the central sulcus. Our results could not be accounted for by differences in the evoked fields, suggesting a unique role of oscillatory activity in empathetic responses. We argue that alpha oscillations provide a unique measure of the underlying functional architecture of the brain, suggesting an automatic disinhibition of the sensorimotor cortices in response to the observation of pain in others.

Keywords: empathy, MEG, pain, mu-rhythm, oscillations, beamformer, sensorimotor, alpha

\section{INTRODUCTION}

When seeing a football player receive a painful tackle we cringe and might even grasp our own knee in affective resonance with the victim's painful state. This phenomenon of vicarious pain experience is explained in the perception-action account of empathy: "The attended perception of the object's state automatically activates the subject's representation of the state, situation, and object, and the activation of these representations automatically primes or generates the associated autonomic and somatic responses, unless inhibited" (Preston and de Waal, 2002).

The perception-action account of empathy for pain has been greatly expanded upon in the last decade, resulting in a nuanced neuroscientific framework that integrates knowledge of affective and perception-action processes with an understanding of the influences of social context, expectation, and attention (for a comprehensive review on its evolutionary basis and social expression in humans and animals see Preston and de Waal, 2002, and for a systematic review on the neuroscience of empathy see Decety and Jackson, 2004; de Vignemont and Singer, 2006; Singer, 2006). de Vignemont and Singer (2006) offer a precise definition of empathy, distinguishing it from cognitive perspective taking on the one hand and emotional contagion on the other. They characterize empathy as being in an affective state isomorphic to another person's affective state, elicited by observation or imagination of another person's affective state, but without losing the understanding that it is the other person's affective state that is the cause of one's own. Two important remarks should be made, however, in light of the current experiment. First of all, the focus on the affective response should not ignore the importance of sensory processes, as we shall see. Secondly, although empathy is certainly not restricted to the sharing of negate affective states, most neuroscientific investigations, this one included, have used the observation of pain as a model to test the notion of shared representations in empathy. Indeed, it has been firmly established that the observation of pain in others involves a network of affective brain regions, such as the anterior cingulate, paracingulate gyrus, and anterior insular, that are also activated during the first-person experience of pain (Ingvar, 1999; Rainville, 2002). Furthermore, responses in these regions have been found using a wide variety of paradigms, from presenting abstract cues of other people in pain (Singer et al., 2004; Jackson et al., 2005; Saarela et al., 2007), to pictures of body parts being pinpricked (Lamm et al., 2007), and painful facial expressions (Jabbi and Keysers, 2008). Empathetic responses in these brain regions have, furthermore, been shown to be influenced by social contexts such as group membership and perceived fairness (Hein et al., 2010) as well as task demands and knowledge about the reality of the stimulus (Gu and Han, 2007). Often, but less consistently, primary somatosensory regions are found to be involved, which seems to depend 
on the availability of the sensory information about the painful stimulus. In their meta-analysis of nine fMRI experiments, Lamm et al. (2011) showed that vicarious activation of the somatosensory cortex seems only to occur when visual details of the flesh-and-bone aspect of the painful situation are observed, not when these are inferred from abstract cues. The authors propose, however, that this somatosensory activation reflects unspecific co-activation elicited by the visual display of body parts rather than a specific matching of the other's somatosensory and nociceptive state. This is in line with their aforementioned characterization of empathy as, first and foremost, an affective state. Other authors, however, have argued for the functional importance of primary sensory cortices as part of the empathetic (pain) response, subserving pain intensity, and location coding (Keysers et al., 2010). Interestingly, in this respect individual propensities differ greatly, as shown by Osborn and Derbyshire (2010). They reported that those people that respond to painful images by experiencing a "real" sensation of pain, show activation of somatosensory cortices, while these regions are not activated in those that do not have such first-person experiences. Further evidence for the involvement of sensory cortices in pain observation comes from electroencephalography (EEG) studies. Bufalari et al. (2007) recorded a reduction of early sensory-evoked potentials after medial nerve stimulation when subjects were watching movies of limbs in painful situations. Pain systems are also tightly linked to action systems (Ingvar, 1999; Saitoh et al., 1999; Juottonen et al., 2002; Farina et al., 2003; Wager et al., 2004), and it has been repeatedly shown that observation of painful movies strongly inhibits corticospinal excitability specific for the muscle that was observed being pinpricked (Avenanti et al., 2005, 2006, 2009). These findings together suggest that primary sensorimotor regions are indeed involved in the empathetic response in ways that entails more than aspecific increases in arousal.

Questions of when empathetic responses occur have been investigated using EEG scalp recordings. Fan and Han (2008) found larger early (140-380 ms) frontal event-related potentials (ERPs) amplitudes in response to pictures of limbs in painful situations compared to those in control situations. These early empathic responses were influenced by contextual reality (real pictures versus cartoons). Later (380-500 ms) central-parietal effects of pain that were prominent in a pain judgment task, were greatly reduced when subjects only had to count the number of limbs. This shows that the empathetic response can be modulated at different times, due to different task contexts, in line with a model of empathy that permits modulation of an automatic perception-action response at multiple stages (de Vignemont and Singer, 2006). Frequency analysis of EEG and magnetoencephalography (MEG) recordings has also been a particularly successful tool in studying the involvement of the sensorimotor system in action, touch, and pain observation. Alpha $(\sim 10 \mathrm{~Hz})$ and beta $(\sim 20 \mathrm{~Hz})$ oscillations originating from the sensorimotor cortex (Hari and Salmelin, 1997) have been implicated in action observation (Hari et al., 1998; Cochin et al., 1999; Jarvelainen et al., 2001; Rossi et al., 2002; Muthukumaraswamy and Johnson, 2004; Muthukumaraswamy et al., 2004; Nakamura et al., 2004; Pineda, 2005; Kilner et al., 2006; Caetano et al., 2007; Oberman et al., 2007; Holz et al., 2008; Koelewijn et al., 2008; van Elk et al., 2008) recognizing point-light biological motion (Ulloa and Pineda, 2007), as well as in understanding communicative gestures (Nakamura et al., 2004). Muthukumaraswamy and Johnson (2004) were the first reporting a reduction of the beta rebound after medial nerve stimulation when subjects concurrently observed a hand being brushed or pricked, but not when only movement was observed. Cheng et al. (2008) also observed reduced alpha rebound after medial nerve stimulation while people watched static pictures of limbs in painful situations. Although these studies point to a modulation of the somatosensory cortex, they do not show how somatosensory oscillations respond to the observation of pain in the absence of actual somatosensory stimulation. To investigate such a visual-to-somatosensory process, three EEG studies investigated effects on ongoing alpha oscillations after observing images of painful situations versus control images, without a contingent transcranial or median nerve stimulation. Two of these studies showed more sensorimotor alpha suppression in response to pain than in response to control images (Yang et al., 2009; Perry et al., 2010), while a third study showed reduced alpha suppression ( $\mathrm{Mu}$ et al., 2008). One reason for these contradictory outcomes might have been the fact that volume conduction makes it difficult to separate sensorimotor alpha (or mu-rhythm) from posterior alpha sources in EEG scalp recordings (Hari and Salmelin, 1997). Since the strongest modulation of alpha power typically involves alphablocking in response to visual stimulation (Pfurtscheller et al., 1996), this activity might have confounded the interpretation of alpha activity from central sources that was found by $\mathrm{Mu}$ et al. (2008). Indeed, Perry et al. (2010) only found increased suppression by pain observation on fronto-central but not on posterior sensors, while Yang et al. (2009) only found increased central alpha suppression. Although these findings strongly suggest alpha suppression in response to the observation of pain in others, they suffer from a lack of spatial resolution needed to univocally establish a sensorimotor origin. These studies also suffered from underspecified or confounded time windows of interest. In Perry et al. (2010) alpha suppression was calculated over the full $2 \mathrm{~s}$ post-stimulus, from stimulus-onset to stimulus offset, while in Yang et al. (2009) the first 1.3 s directly after stimulus-onset were used. In both cases stimulus-onset evoked responses were therefore included, making it ambiguous to what degree their observations can be interpreted exclusively as a modulation of ongoing alpha activity and to what degree evoked responses contributed (Steriade et al., 1990; Lopes da Silva, 1991; Pfurtscheller and Lopes da Silva, 1999; Mazaheri and Jensen, 2010). Mu et al. (2008) did perform an analysis on separate time windows and reported modulation of the alpha suppression only between 200 and $400 \mathrm{~ms}$ after stimulus-onset. They also tried to minimize the effect of phase-locked activity on the power estimate by subtracting average ERP in response to stimulus-onset. Since images were presented for only $200 \mathrm{~ms}$, the power estimation might have still been confounded by transients in response to stimulus offset. Finally, the evaluation of every stimulus in $\mathrm{Mu}$ et al. (2008) might have resulted in motor-preparation which previously has been shown to interact with sensorimotor oscillations in response to pain (Babiloni et al., 2008).

Concerns about the mixing of central and posterior sources, evoked activity and motor-preparation aside, the differences in the direction of alpha modulation might also point to the interesting possibility that different functional processes were involved. Historically, alpha activity has been interpreted as reflecting a 
non-functional "cortical idling" state (Pfurtscheller et al., 1996). This view has recently been challenged, and a more functional interpretation of alpha has been formulated that describes a mechanisms of gating-through-inhibition (Klimesch, 1999; Neuper and Pfurtscheller, 2001; Schack and Klimesch, 2002; Jensen and Mazaheri, 2010). According to this view, task-irrelevant regions are inhibited through an increase of alpha oscillations, routing information to task-relevant regions. For instance, it has been demonstrated that alpha activity over visual areas increases in motor tasks and vice versa (Pfurtscheller et al., 1996). A similar mechanism seems to function when attention is directed within the visual or somatosensory domain. For instance, when covert attention is directed to one hemifield (e.g., the left), alpha decreases in the contralateral (right) hemisphere but increases in the ipsilateral (left) hemisphere (Worden et al., 2000; Thut et al., 2006; Rihs et al., 2007; Kelly et al., 2009; van Gerven and Jensen, 2009; Handel et al., 2010). Alpha activity was also shown to decrease in the primary sensorimotor cortex contralateral to the engaged hand while it increased in the ipsilateral hemisphere during a somatosensory working memory task. In line with such a view, we propose that the findings of Yang et al. (2009) and Perry et al. (2010) show how observation of pain in others induces a disinhibition of the somatosensory cortex through alpha suppression. This would create the optimal cortical context in which somatosensory processes such as location and intensity coding of the observed pain (Keysers et al., 2010) can be performed. Such an account might also tentatively explain the increase in alpha reported by $\mathrm{Mu}$ et al. (2008). The short (200 ms) presentation of images, together with the task of evaluating these on their painful content, could have resulted in an increase of functional inhibition of the somatosensory cortices for the purpose of reducing interference during the evaluation of the somatosensory (pain) representation. Similar processes have indeed been shown during the retention interval in a visual long term memory task (Meeuwissen et al., in press) as well as during as somatosensory working memory task (Haegens et al., 2011) where distraction in the visual or somatosensory modality was inhibited.

In the current experiment all of the previous concerns were dealt with for the purpose of unequivocally identifying sensorimotor alpha suppression in the observation of pain in others. Subjects viewed images of limbs in pain and no-pain situations in a passive task that required no evaluation or motor responses to the stimuli. To reduce concerns of mixing sources from different regions we recorded brain activity using MEG and applied a beamformer technique for source estimation. We did a time-frequency analysis over the whole post-stimulus interval, but restricted our statistical analysis on the non-evoked period (>400 ms). We hypothesized greater sensorimotor alpha suppression in response to painful images than in response to the observation of control images.

\section{MATERIALS AND METHODS PARTICIPANTS}

Twenty-one healthy participants ( 15 female, mean age 26.6 years, range: 20-49) enrolled after providing written informed consent and were paid in accordance with guidelines of the local ethics committee (CMO Committee on Research Involving Humans subjects, region Arnhem-Nijmegen, the Netherlands). Two participants were excluded from the analysis due to excessive eye or movement artifacts. One subject fell asleep during the experiment and was excluded as well. The experiment was in compliance with national legislation as well as the code of ethical principles (declaration of Helsinki).

\section{STIMULI}

A series of 128 digital color pictures showing right hands and right feet in painful and non-painful situations were used. These stimuli were previously used and validated in behavioral and fMRI studies (Jackson et al., 2005, 2006) and one MEG study (Cheng et al., 2008). All pictures depicted familiar events that can happen in everyday life involving mechanical, thermal, and pressure pain. The neutral pictures involved the same settings without any painful component. All pictures were edited to the same size $(600 \times 450$ pixels $)$.

\section{SUBJECTIVE EMPATHY INDEX}

Within 2 weeks before the experiment commenced subjects filled in the Interpersonal Reactivity Index, a self-report questionnaire measuring different factors related to empathy (Davis, 1983).

\section{EXPERIMENT}

While seated in the MEG system, the stimuli were projected on a screen about $80 \mathrm{~cm}$ in front of the subject. These were all presented in random order for $1.5 \mathrm{~s}$ per trial, interleaved with gray fixations screens of $1.5 \mathrm{~s}$ (Figure 1). The procedure was repeated over three

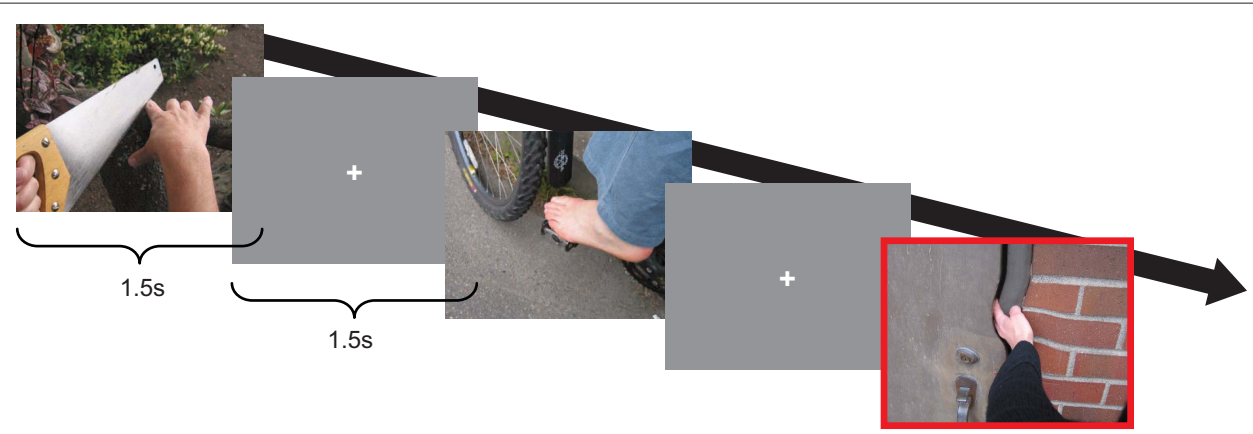

FIGURE 1 |The experimental paradigm. Subjects were presented with pictures depicting limbs painful and non-painful situation, interleaved with gray fixation screens. $10 \%$ of the pictures showed a small rotation in the center of the picture (red highlight), the total number of which they had to internally count and report back after each session. 
blocks resulting in a total experimental time of $\sim 45 \mathrm{~min}$. Subjects were instructed to remain relaxed and not move their limbs, their compliance observed by the experimenter using infrared camera. To make sure subjects paid attention to the stimuli, 10 percent of presentations showed a short twisted movement, created by shortly ( $\sim 500 \mathrm{~ms})$ presenting, within one stimulation, the same picture modified with a twirl filter (Photoshop, Adobe Systems Inc.). Subjects were required to internally count the number of these occurrences and report them to the experimenter after each block. Target stimuli were discarded from further analysis. The experiment was programmed and ran using the software package Presentation ${ }^{1}$.

\section{DATA ACQUISITION}

Continuous MEG was recorded using a 275 sensor axial gradiometer system (CTF MEG TM Systems Inc., Port Coquitlam, BC, Canada) placed in a magnetically shielded room. The ongoing MEG signals were low-pass filtered at $300 \mathrm{~Hz}$, digitized at $1200 \mathrm{~Hz}$, and stored for off-line analysis. The subjects' head position was continuously recorded relative to the gradiometer array using coils positioned at the subject's nasion and at the left and right ear canals. High-resolution anatomical images $(1 \mathrm{~mm}$ isometric voxel size) were acquired using a 1.5-T Siemens Magnetom Sonata system (Erlangen, Germany). The same earplugs, using vitamin E instead of the coils, were used for coregistration with the MEG data.

\section{DATA ANALYSIS}

Magnetoencephalography data was analyzed using the Matlabbased Fieldtrip toolbox, developed at the Donders Institute for Brain, Cognition and Behavior (Oostenveld et al., 2011). Trials containing movement, muscle, and superconducting quantum interference device (SQUID) jumps were discarded by visual inspection. Independent component analysis (ICA) was used to remove eye and heart artifacts. For the sensor-level analysis, planar gradients of the MEG field distribution were calculated using a nearest-neighbor method comparable with the method described by Bastiaansen and Knosche (2000) and also applied by, e.g., Jokisch and Jensen (2007), Nieuwenhuis et al. (2008), Mazaheri et al. (2009), Haegens et al. (2010), and Haegens et al. (2011). The horizontal and vertical components of the estimated planar gradients approximate the signal measured by planar gradiometers while making the sensorlevel data easier to interpret as the maximal activity is typically located above the source (Hamalainen et al., 1993). For source reconstruction, however, we used the original data from the axial sensors.

\section{TIME-FREQUENCY AND ERF ANALYSIS ON THE SENSOR LEVEL}

For the time window surrounding the stimulus ( -0.4 to $1.5 \mathrm{~s})$, time-frequency representations (TFRs) of power were calculated using a Hanning taper approach applied to short sliding time windows (Percival and Walden, 1993) using an adaptive time window of four cycles length $(\Delta t=4 / f)$. The data in each time window were multiplied with a Hanning taper. The power

${ }^{1}$ http://nbs/neurobs.com values were calculated as the sum of the horizontal and vertical component of the estimated planar gradient after subtracting the mean amplitude from the entire time interval. The planar gradient power estimates were subsequently averaged over trials for the pain and control condition. To investigate the event-related changes in activity we calculated the change of power in response to stimulus presentation relative to the average power during the 200-ms before stimulus-onset.

For the time window surrounding the stimulus ( -0.4 to $1.5 \mathrm{~s})$, event-related fields (ERFs) were calculated. The data were then low-pass filtered at $40 \mathrm{~Hz}$ using a butterworth filter (order of six), and averaged separately for every condition. Similarly as with the frequency analysis, averaging was done on the planar gradients after which they were summed.

\section{STATISTICAL ANALYSIS ON THE SENSOR LEVEL}

To avoid "double dipping" (Kriegeskorte et al., 2009) we restricted our statistical comparison to those sensors where we have previously shown the central mu-rhythm to be maximally modulated in a somatosensory task (Haegens et al., 2011: MLC24, MLC25, MLC31, MLC32, MLP35, MLC42, MLP23, MRC24, MRC25, MRC31, MRC32, MRP35, MRC42, MRP23, see highlighted sensors in Figure 3A); We compared the mean log transformed alpha $(7-14 \mathrm{~Hz})$ and beta $(15-25 \mathrm{~Hz})$ band power over $1 \mathrm{~s}$ post-stimulus period, starting at $400 \mathrm{~ms}$ to exclude the contribution of evoked components. Although no differences in the beta-band activity were expected, a clear beta suppression in response to both pain and non-painful stimuli prompted an ad hoc testing for differences between conditions.

\section{SOURCE RECONSTRUCTION}

Source reconstruction was performed using a frequency-domain beamformer approach (Dynamic Imaging of Coherent Sources) which uses adaptive spatial filters to localize power in the entire brain (Gross et al., 2001; Liljestrom et al., 2005). The brain volume of each individual subject was discretized to a grid with a $0.8-\mathrm{cm}$ resolution. For every grid point a spatial filter was constructed from the cross-spectral density matrix and the lead field. The lead fields were calculated from a subject specific realistic single-shell model of the brain (Nolte, 2003), based on the individual anatomical MRIs. We calculated the cross-spectral density matrix based upon both the post-stimulus (200-1400 ms) as well as pre-stimulus (1400-200 ms pre-stimulus) interval to obtain the most accurate estimation of the alpha source. Furthermore, both conditions were combined for the purpose of calculating the spatial filter, after which the power at each grid point was estimated for both conditions separately in every subject. Sources were estimated using a multitaper approach to accomplish accurate frequency smoothing for the alpha band ( $10 \pm 2 \mathrm{~Hz}$ by using three Slepian tapers). Prior to averaging, the source estimates of the individual subjects' functional data were spatially normalized using SPM2 to the International Consortium for Brain Mapping template (Montreal Neurological Institute, MNI, Montreal, QC, Canada $\left.^{2}\right)$.

${ }^{2} \mathrm{http}: / /$ www.bic.mni.mcgill.ca/brainweb 


\section{RESULTS}

Subjects were presented with static images depicting limbs in painful and non-painful situations from a first-person perspective. We investigated the role of sensorimotor rhythms in processing the painful content.

\section{WIDE-SPREAD MODULATION OF ALPHA- AND BETA-BAND ACTIVITY FOLLOWING VISUAL STIMULI}

First, we investigated the responses to combined painful and nonpainful stimuli. As shown in Figure 2A, these resulted in marked reductions in occipital alpha (mean $49.9 \%$ of baseline, SEM 5.3\%), extending to central sensors power (mean $74 \%$ of baseline, SEM $4.3 \%$ ). At central sensors separate alpha and beta components could readily be distinguished (Figures 2B,C). This demonstrates widespread modulation in the alpha and beta frequencies including both occipital and central regions.

\section{GREATER SENSORIMOTOR ALPHA SUPPRESSION IN RESPONSE TO PAINFUL PICTURES}

We then tested if these oscillatory responses were different in response to painful compared to non-painful stimuli. As predicted, the strongest modulation was observed at central regions over the head (Figure 3A), showing consistency in topography with a previous investigation of attention-related alpha modulation using the same MEG system and comparable spectral analysis (Haegens et al., 2011). A $t$-test comparing the average (log transformed) power between pain and control pictures on these sensors (Figure 3B) yielded a significant difference (mean difference 2.19\%,
SEM $=0.63 \%, p=0.019$, one-sided) demonstrating that the painful stimuli resulted in stronger sensorimotor alpha suppression than pictures showing non-painful situations. Difference in beta power only showed a trend toward increased suppression (mean difference $1.41 \%, \mathrm{SEM}=1.07 \%, p=0.062$, one-sided). Source analysis confirmed that the origin of the alpha difference was located along the central sulcus (Figure 3D).

\section{NO DIFFERENCE IN THE ERF BETWEEN PAINFUL AND CONTROL PICTURES}

We also calculated the ERFs to investigate if pain observation could be observed in neuronal activity time-locked to the stimulus. Early visual evoked components $(<400 \mathrm{~ms})$ were clearly reflected in the ERFs, while during the interval in which we found sustained alpha suppression $(>400 \mathrm{~ms})$ the ERF deflection returned toward baseline. A cluster-based randomization test based upon every timepoint found no difference between conditions for neither occipital nor central sensors (depicted in the boxes of Figure 2A), nor for the central sensors selected for our frequency analysis (Figure 3C). Our findings of increased alpha suppression therefore seem unrelated to differences in evoked responses.

\section{NO CORRELATIONS WITH SUBJECTIVE EMPATHY REPORTS}

We also tested for correlations between the magnitude of the modulation of the neuronal response by the pain effect and subjective reports of empathetic distress in daily life. Individual scores on the Interpersonal Reactivity Index (Davis, 1983) did not correlate reliably with the magnitude of the alpha modulation (perspective

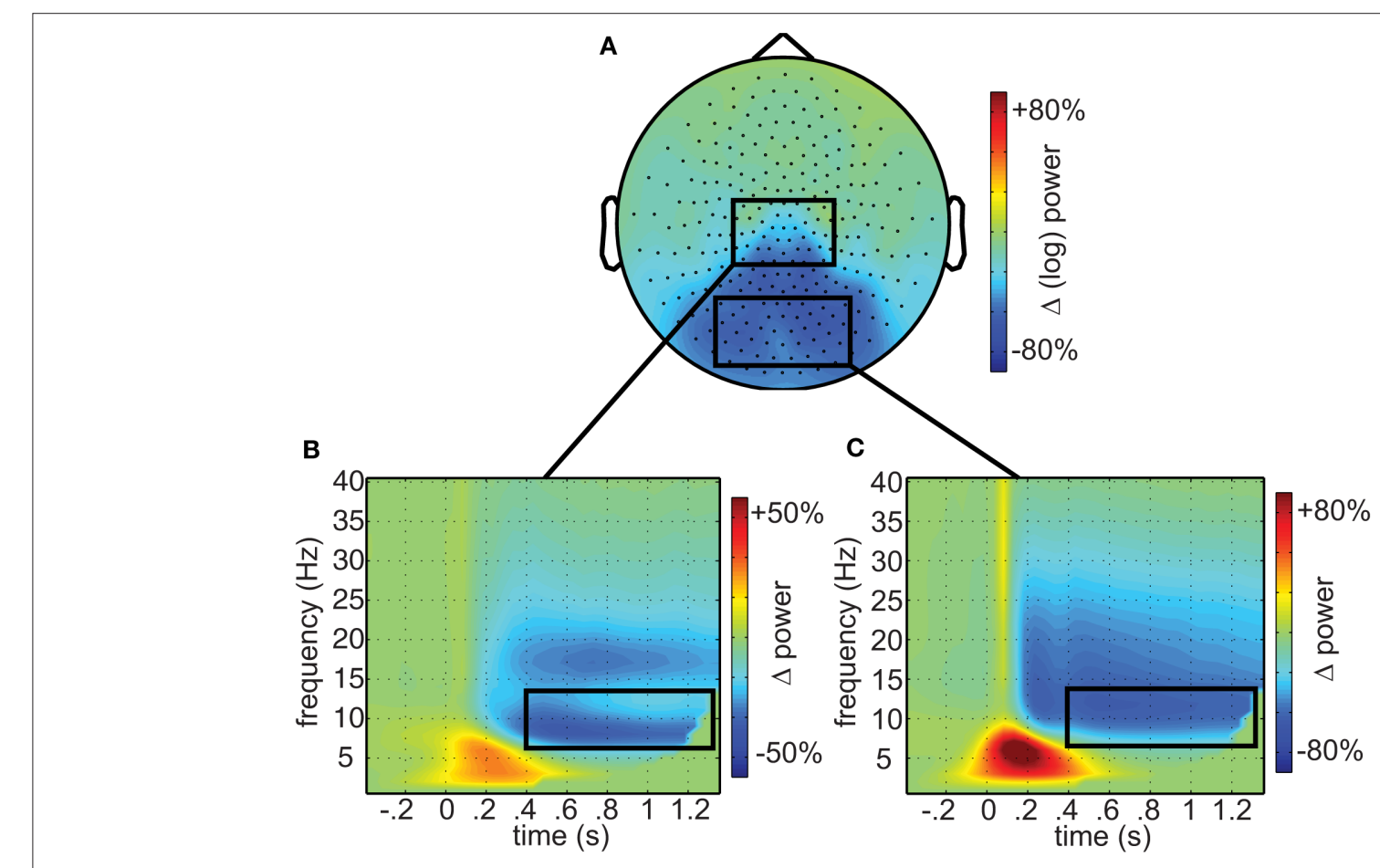

FIGURE 2 | Oscillatory responses to visual stimuli. (A) The topographic representation of alpha (7-14 Hz) suppression in response to visual stimuli (0.5-1.35 s). (B) Time-frequency representations of central and (C) occipital sensors, respectively, as identified by the boxes in (A). Time-frequency window of interest (0.4-1.35 s; 7-14 Hz) outlined in black. 
A

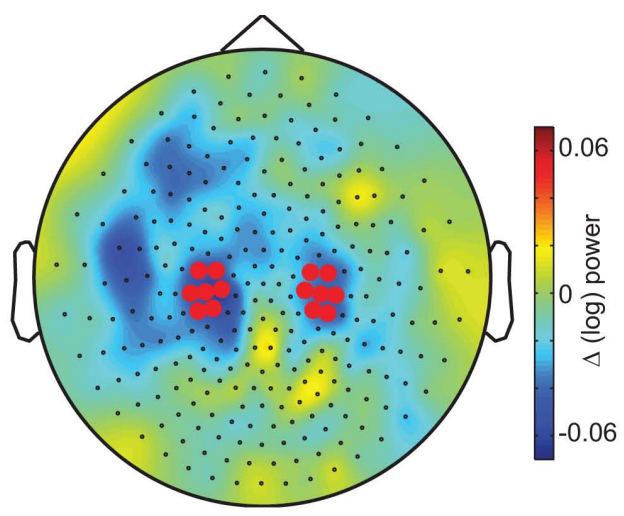

C

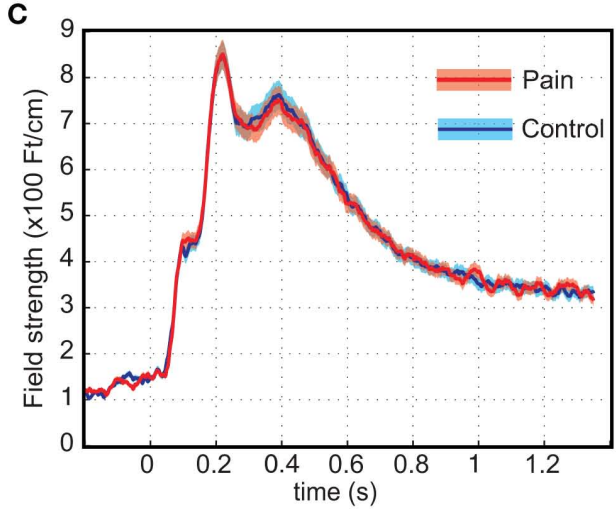

FIGURE 3 | Sensorimotor alpha suppression in response to pain. (A)The topographical representation of the difference in sensorimotor alpha power (7-14 Hz) calculated by subtracting average alpha power $(0.4-1.35 \mathrm{~s}$; log transformed) of the no-pain stimuli from the painful stimuli. Highlighted sensors are taken from Haegens et al. (2011). (B) Time-frequency representation of the

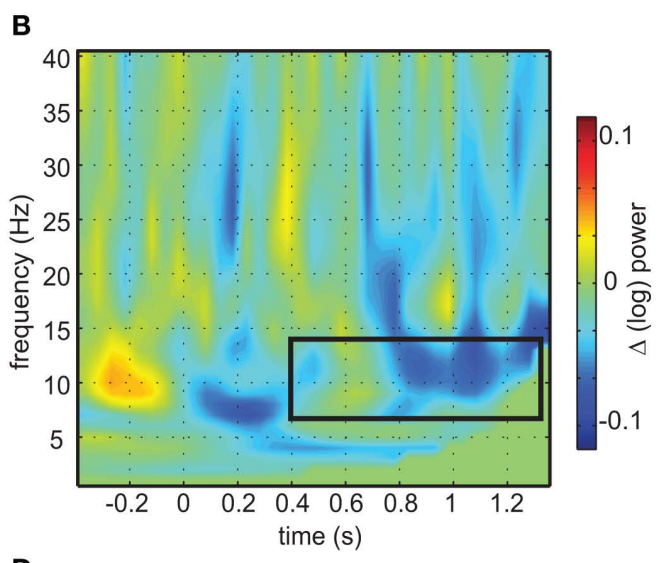

D

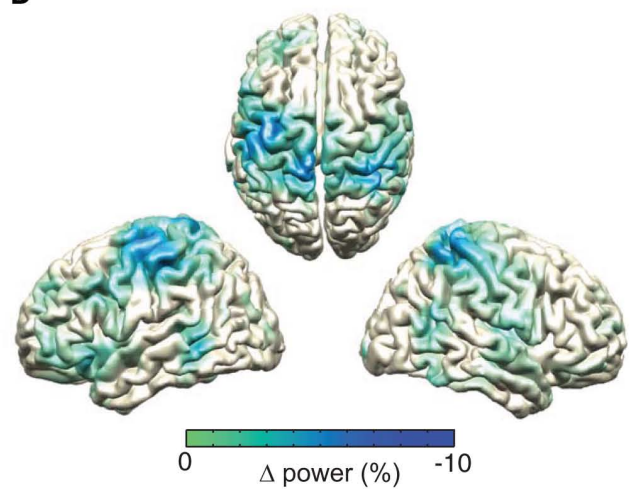

pain minus no-pain condition of the highlighted sensors in (A). Box depicts time-frequency window of interest $(p=0.019)$. (C) Evoked responses and SD for pain and no-pain averaged over highlighted sensors from (A). (D) Source reconstructions of alpha difference obtained using beamformer, showing sources along the central sulcus. taking: $p=0.494$, empathetic concern: $p=0.862$; fantasy: $p=0.433$; personal distress: $p=0.248$; total: $p=0.522$ ), nor with the magnitude of the beta modulation (perspective taking: $p=0.667$, empathetic concern: $p=0.910$; fantasy: $p=0.829$; personal distress: $p=0.486$; total: $p=0.960)$.

\section{DISCUSSION}

We used MEG to investigate neural oscillations in vicarious pain perception. We found wide-spread alpha- and beta-band depression in response to visual stimuli, predominantly at posterior sensors. In contrast to these wide-spread visual responses, observing pain depressed alpha power selectively more at central sensors. By applying source modeling we identified the sources of this difference along the central sulcus, implicating sensorimotor regions in the observation of pain. Strikingly, central and posterior ERFs did not show differences between conditions, suggesting a unique role for induced activity in the brain's response to observing other people in pain. These results provide support for the involvement of sensorimotor oscillations in empathetic responses.

Alpha oscillations might be providing a graded level of excitability and inhibition in task relevant and irrelevant regions, streamlining information flow dependent on moment-by-moment task demands (Klimesch, 1999; Neuper and Pfurtscheller, 2001; Schack and Klimesch, 2002; Jensen and Mazaheri, 2010). Increased alpha suppression in the observation of pain is also in accordance with previous reports of oscillatory involvement in the subjective perception of (first-person) pain intensity (Babiloni et al., 2006) and in the anticipation of pain (Babiloni et al., 2008) and fits well within the general understanding that sensorimotor alpha oscillations provide a dynamically modulated cortical context for somatosensory processing (Lopes da Silva, 1991; Pfurtscheller and Lopes da Silva, 1999; Haegens et al., 2010, 2011). What sensorimotor alpha suppression might represent in terms of underlying empathetic mechanisms remains under debate, however. While activation of sensorimotor regions in fMRI studies can be interpreted in terms of perceptionaction coupling (Keysers et al., 2010), or of unspecific co-activation (Lamm et al., 2011), our results taken together with the emerging understanding of the functional relevance of alpha oscillations (Lopes da Silva, 1991; Klimesch, 1999; Jensen and Mazaheri, 2010), however strongly suggest a disinhibition of the sensorimotor cortices in response to the observation of pain in others. It is in no way suggested, however, that sensorimotor alpha suppression is by itself sufficient for empathy. As has been argued convincingly at length elsewhere (de Vignemont and Singer, 2006; Keysers et al., 2010; 
Lamm et al., 2011), empathy is a multidimensional response to a variety of situations, real and imagined. Not one neural mechanism will be able to explain its full expression. Noteworthy are the results by Betti et al. (2009) who found increased gamma band coherence between primary sensory and motor regions when subjects watched painful movies. Their results further emphasize the importance of understanding empathy through dynamic oscillatory interactions of neuronal assemblies distributed within and across different specialized brain regions. Neither is it claimed here that sensorimotor alpha oscillations are specifically involved in social processes. We understand alpha oscillations more generally as a (sensory) mechanism for gating-through-inhibition, which, in this particular case, is involved in the task of disinhibiting sensory cortices in response to the observation of pain in others.

Although we were able to localize the source of the empathetic alpha modulation along the central sulcus, we were not able to distinguish between primary somatosensory from primary motor regions. This might have been a problem of lack of signal strength, compared to those studies that have been able to localized alpha modulation to postcentral sulcus in response to actual tactile stimulation. For instance, in Caetano et al. (2007) observation of movement resulted in only $42 \%$ of the beta suppression that was found in response to actual movement. As we localized the difference between the pain and no-pain condition, a difference of only a couple of percent, this problem might have been exacerbated even further.

The fact that this study is limited to the observation of limbs might limit a generalization to the observation of other body parts that afford less action related responses. In fact, as mentioned before, besides the fact that sensorimotor alpha is clearly implicated in somatosensory attention and perception, it has also been a hallmark of action-perception. In our study the amount of action suggested in the images was, however, kept similar between conditions. For instance, where in the control condition one situation depicts the sawing through of a wooden log, the painful counterpart differed only by the fact that the second hand was in a painful position underneath the saw. Any difference between the observation of painful and control images therefore cannot be explained by a difference in action-perception between these conditions. This fact, together with the fact that alpha oscillations are commonly found to have a postcentral source (Salmelin and Hari, 1994; Cheyne et al., 2003; Jurkiewicz et al., 2006) makes an interpretation of our findings in terms of motor processes less likely.

Although subjects were instructed to remain relaxed and not move their limbs, and no responses were required, this cannot fully rule out "covert movement" or muscle tension in response to the

\section{REFERENCES}

Avenanti, A., Bueti, D., Galati, G., and Aglioti, S. M. (2005). Transcranial magnetic stimulation highlights the sensorimotor side of empathy for pain. Nat. Neurosci. 8, 955-960.

Avenanti, A., Minio-Paluello, I., Bufalari, I., and Aglioti, S. M. (2006). Stimulusdriven modulation of motor-evoked potentials during observation of others' pain. Neuroimage 32, 316-324.

Avenanti, A., Minio-Paluello, I., Bufalari, I., and Aglioti, S. M. (2009). The pain of a

painful images. Although some (e.g., Yang et al., 2009, but not Perry et al., 2010) record electromyography (EMG), a null-finding comparing average EMG across conditions will not be able to fully rule out differences below the sensitivity of the measurement, and any selection of muscles would have its limitations. More importantly, we did not find significant differences in the beta $(\sim 20 \mathrm{~Hz})$ band where effects of movement would be most pronounced (Salmelin et al., 1995; Hari and Salmelin, 1997; Cheyne et al., 2003; Jurkiewicz et al., 2006; Parkes et al., 2006).

We did not find any correlation with self-report questionnaires and contribute to the existing confusion in the literature. In Fan and Han (2008), self-reported unpleasantness of the observed pain stimuli correlated with early evoked potentials to painful observation, but did so only in an evaluation task and not in the counting task, which would be more similar to our passive paradigm. Mu et al. (2008) reported negative correlations for both perceived pain and self-unpleasantness, also in a similar evaluation task. Conversely, Cheng et al. (2008) found only positive correlations of perspective taking with sensory-evoked alpha rebound, and no correlation with empathy quotient questionnaire (EQ: BaronCohen and Wheelwright, 2004, the emotional contagion scale, ECS: Doherty, 1997) or any subscales of the interpersonal reaction index (IRI: Davis, 1983). Finally, Yang et al. (2009) found that the effect of pain on the alpha rebound correlated with the personal distress subscale of the IRI, but only in females. Taken together, it seems that the most consistent findings have been found correlating the subjective quality of individual stimuli trials with oscillatory activity, while correlating individual traits have been less conclusive.

Concluding, observing limbs in pain suppressed ongoing alpha oscillations more than observing control images. This effect was localized along the central sulcus, implicating somatosensory alpha oscillations in the observation of pain in others. These findings support an interpretation in terms of gating-through-inhibition where observation of painful images disinhibits the somatosensory cortex through alpha suppression.

\section{ACKNOWLEDGMENTS}

The authors gratefully acknowledge the support of the BrainGain Smart Mix Programme of the Netherlands Ministry of Economic Affairs and the Netherlands Ministry of Education, Culture and Science the Netherlands Initiative Brain and Cognition, a part of the Organization for Scientific Research (NWO) under grant number SSM0611 and the Netherlands Organization for Scientific Research (NWO): Innovational Research Incentive Schemes, VICI grant number: 453-09-002.

M., Cibelli, G., Romani, G. L., Rossini, P. M., and Arendt-Nielsen, L. (2008). Cortical alpha rhythms are related to the anticipation of sensorimotor interaction between painful stimuli and movements: a high-resolution EEG study. J. Pain 9, 902-911.

Baron-Cohen, S., and Wheelwright, S. (2004). The empathy quotient: an investigation of adults with Asperger syndrome or high functioning autism, and normal sex differences. J. Autism Dev. Disord. 34, 163-175.
Bastiaansen, M. C., and Knosche, T. R. (2000). Tangential derivative mapping of axial MEG applied to eventrelated desynchronization research. Clin. Neurophysiol. 111, 1300-1305.

Betti, V., Zappasodi, F., Rossini, P. M., Aglioti, S. M., and Tecchio, F. (2009). Synchronous with your feelings: sensorimotor \{gamma\} band and empathy for pain. J. Neurosci. 29, 12384-12392.

Bufalari, I., Aprile, T., Avenanti, A., Di Russo, F., and Aglioti, S. M. (2007). 
Empathy for pain and touch in the human somatosensory cortex. Cereb. Cortex 17, 2553-2561.

Caetano, G., Jousmaki, V., and Hari, R. (2007).Actor's and observer's primary motor cortices stabilize similarly after seen or heard motor actions. Proc. Natl. Acad. Sci. U.S.A. 104, 9058-9062.

Cheng, Y., Yang, C. Y., Lin, C. P., Lee, P. L., and Decety, J. (2008). The perception of pain in others suppresses somatosensory oscillations: a magnetoencephalography study.Neuroimage 40, 1833-1840.

Cheyne, D., Gaetz, W., Garnero, L., Lachaux, J. P., Ducorps, A., Schwartz, D., and Varela, F. J. (2003). Neuromagnetic imaging of cortical oscillations accompanying tactile stimulation. Brain Res. Cogn. Brain Res. 17, 599-611.

Cochin, S., Barthelemy, C., Roux, S., and Martineau, J. (1999). Observation and execution of movement: similarities demonstrated by quantified electroencephalography. Eur. J. Neurosci. 11, 1839-1842.

Davis, M. H. (1983). Measuring individual-differences in empathy - evidence for a multidimensional approach. $J$. Pers. Soc. Psychol. 44, 113-126.

de Vignemont, F., and Singer, T. (2006). The empathic brain: how, when and why? Trends Cogn. Sci. 10, 435-441.

Decety, J., and Jackson, P. L. (2004). The functional architecture of human empathy. Behav. Cogn. Neurosci. Rev. 3, 71-100.

Doherty, R. W. (1997). The emotional contagion scale: a measure of individual differences. J. Nonverbal Behav. 21, 131-154.

Fan, Y., and Han, S. (2008). Temporal dynamic of neural mechanisms involved in empathy for pain: an event-related brain potential study. Neuropsychologia 46, 160-173.

Farina, S., Tinazzi, M., Le Pera, D., and Valeriani, M. (2003). Pain-related modulation of the human motor cortex. Neurol. Res. 25, 130-142.

Gross, J., Kujala, J., Hamalainen, M., Timmermann, L., Schnitzler, A., and Salmelin, R. (2001). Dynamic imaging of coherent sources: studying neural interactions in the human brain. Proc. Natl. Acad. Sci. U.S.A. 98, 694-699.

Gu, X., and Han, S. (2007). Attention and reality constraints on the neural processes of empathy for pain. Neuroimage 36, 256-267.

Haegens, S., Handel, B. F., and Jensen, O. (2011). Top-down controlled alpha band activity in somatosensory areas determines behavioral performance in a discrimination task. J. Neurosci. 31, 5197-5204.

Haegens, S., Osipova, D., Oostenveld, R., and Jensen, O. (2010). Somatosensory working memory performance in humans depends on both engagement and disengagement of regions in a distributed network. Hum. Brain Mapp. 31, 26-35.

Hamalainen, M., Hari, R., Ilmoniemi, R. J., Knuutila, J., and Lounasmaa, O. V. (1993). Magnetoencephalography theory, instrumentation, and applications to noninvasive studies of the working human brain. Rev. Mod. Phys. 65, 413-497.

Handel, B. F., Haarmeier, T., and Jensen, O. (2010). Alpha oscillations correlate with the successful inhibition of unattended stimuli. J. Cogn. Neurosci.

Hari, R., Forss, N., Avikainen, S., Kirveskari, E., Salenius, S., and Rizzolatti, G. (1998). Activation of human primary motor cortex during action observation: a neuromagnetic study. Proc. Natl. Acad. Sci. U.S.A. 95, 15061-15065.

Hari, R., and Salmelin, R. (1997). Human cortical oscillations: a neuromagnetic view through the skull. Trends Neurosci. 20, 44-49.

Hein, G., Silani, G., Preuschoff, K., Batson, C. D., and Singer, T. (2010). Neural responses to ingroup and outgroup members' suffering predict individual differences in costly helping. Neuron 68, 149-160.

Holz, E. M., Doppelmayr, M., Klimesch, W., and Sauseng, P. (2008). EEG correlates of action observation in humans. Brain Topogr. 21, 93-99.

Ingvar, M. (1999). Pain and functional imaging. Philos. Trans. R. Soc. Lond. B Biol. Sci. 354, 1347-1358.

Jabbi, M., and Keysers, C. (2008). Inferior frontal gyrus activity triggers anterior insula response to emotional facial expressions. Emotion 8, 775-780.

Jackson, P. L., Meltzoff, A. N., and Decety, J. (2005). How do we perceive the pain of others? A window into the neural processes involved in empathy. Neuroimage 24, 771-779.

Jackson, P. L., Rainville, P., and Decety, J. (2006). To what extent do we share the pain of others? Insight from the neural bases of pain empathy. Pain 125, 5-9. Jarvelainen, J., Schurmann, M., Avikainen, S., and Hari, R. (2001). Stronger reactivity of the human primary motor cortex during observation of live rather than video motor acts. Neuroreport 12, 3493-3495.

Jensen, O., and Mazaheri, A. (2010). Shaping functional architecture by oscillatory alpha activity: gating by inhibition. Front. Hum. Neurosci. 4:186. doi: 10.3389/fnhum.2010.00186 Jokisch, D., and Jensen, O. (2007). Modulation of gamma and alpha activity during a working memory task engaging the dorsal or ventral stream. J. Neurosci. 27, 3244-3251.
Juottonen, K., Gockel, M., Silen, T., Hurri, H., Hari, R., and Forss, N. (2002). Altered central sensorimotor processing in patients with complex regional pain syndrome. Pain 98, 315-323.

Jurkiewicz, M. T., Gaetz, W. C., Bostan, A. C., and Cheyne, D. (2006). Postmovement beta rebound is generated in motor cortex: evidence from neuromagnetic recordings. Neuroimage 32, 1281-1289.

Kelly, S. P., Gomez-Ramirez, M., and Foxe, J. J. (2009). The strength of anticipatory spatial biasing predicts target discrimination at attended locations: a high-density EEG study. Eur. J. Neurosci. 30, 2224-2234.

Keysers, C., Kaas, J. H., and Gazzola, V. (2010). Somatosensation in social perception. Nat. Rev. Neurosci. 11, 417-428.

Kilner, J. M., Marchant, J. L., and Frith, C. D. (2006). Modulation of the mirror system by social relevance. Soc. Cogn. Affect. Neurosci. 1, 143-148.

Klimesch, W. (1999). EEG alpha and theta oscillations reflect cognitive and memory performance: a review and analysis. Brain Res. Brain Res. Rev. 29, 169-195.

Koelewijn, T., Van Schie, H. T., Bekkering, $\mathrm{H}$., Oostenveld, R., and Jensen, $\mathrm{O}$. (2008). Motor-cortical beta oscillations are modulated by correctness of observed action. Neuroimage 40, 767-775.

Kriegeskorte, N., Simmons, W. K. Bellgowan, P. S., and Baker, C. I. (2009). Circular analysis in systems neuroscience: the dangers of double dipping. Nat. Neurosci. 12, 535-540.

Lamm, C., Decety, J., and Singer, T. (2011). Meta-analytic evidence for common and distinct neural networks associated with directly experienced pain and empathy for pain. Neuroimage 54, 2492-2502.

Lamm, C., Nusbaum, H. C., Meltzoff, A. N., and Decety, J. (2007). What are you feeling? Using functional magnetic resonance imaging to assess the modulation of sensory and affective responses during empathy for pain. PLoS ONE 2, e1292. doi: 10.1371/ journal.pone.0001292

Liljestrom, M., Kujala, J., Jensen, O., and Salmelin, R. (2005). Neuromagnetic localization of rhythmic activity in the human brain: a comparison of three methods. Neuroimage 25, 734-745.

Lopes da Silva, F. (1991). Neural mechanisms underlying brain waves: from neural membranes to networks. Electroencephalogr. Clin. Neurophysiol. 79, 81-93.

Mazaheri, A., and Jensen, O. (2010). Rhythmic pulsing: linking ongoing brain activity with evoked responses.
Front. Hum. Neurosci. 4:177. doi: 10.3389/fnhum.2010.00177

Mazaheri, A., Nieuwenhuis, I. L., Van Dijk, H., and Jensen, O. (2009). Prestimulus alpha and mu activity predicts failure to inhibit motor responses. Hum. Brain Mapp. 30, 1791-1800.

Meeuwissen, E. B., Takashima, A., Fernandez, G., and Jensen, O. (in press). Increase in posterior alpha activity during rehearsal predicts successful long-term memory formation of word sequences. Hum Brain Mapp. doi: 10.3389/fnhum.2010.00186

Mu, Y., Fan, Y., Mao, L., and Han, S. (2008). Event-related theta and alpha oscillations mediate empathy for pain. Brain Res. 1234, 128-136.

Muthukumaraswamy, S. D., and Johnson, B. W. (2004). Primary motor cortex activation during action observation revealed by wavelet analysis of the EEG. Clin. Neurophysiol. 115, 1760-1766.

Muthukumaraswamy, S. D., Johnson, B. W., and Mcnair, N. A. (2004). Mu rhythm modulation during observation of an object-directed grasp. Brain Res. Cogn. Brain Res. 19, 195-201.

Nakamura, A., Maess, B., Knosche, T. R., Gunter, T. C., Bach, P., and Friederici, A. D. (2004). Cooperation of different neuronal systems during hand sign recognition. Neuroimage 23, 25-34.

Neuper, C., and Pfurtscheller, G. (2001). Event-related dynamics of cortical rhythms: frequency-specific features and functional correlates. Int. J. Psychophysiol. 43, 41-58.

Nieuwenhuis, I. L., Takashima, A., Oostenveld, R., Fernandez, G., and Jensen, O. (2008). Visual areas become less engaged in associative recall following memory stabilization. Neuroimage 40, 1319-1327.

Nolte, G. (2003). The magnetic lead field theorem in the quasi-static approximation and its use for magnetoencephalography forward calculation in realistic volume conductors. Phys. Med. Biol. 48, 3637-3652.

Oberman, L. M., Pineda, J. A., and Ramachandran, V. S. (2007). The human mirror neuron system: a link between action observation and social skills. Soc. Cogn. Affect. Neurosci. 2, 62-66.

Oostenveld, R., Fries, P., Maris, E., and Schoffelen, J. M. (2011). FieldTrip: open source software for advanced analysis of MEG, EEG, and invasive electrophysiological data. Comput. Intell. Neurosci. 2011. doi: 10.1155/2011/156869

Osborn, J., and Derbyshire, S. W. (2010). Pain sensation evoked by observing injury in others. Pain 148, 268-274.

Parkes, L. M., Bastiaansen, M. C., and Norris, D. G. (2006). Combining 
EEG and fMRI to investigate the postmovement beta rebound. Neuroimage 29, 685-696.

Percival, D. B., and Walden, A. T. (1993). Spectral Analysis for Physical Applications: Multitaper and Conventional Univariate Techniques. New York: Cambridge University Press.

Perry, A., Bentin, S., Bartal, I. B., Lamm, C., and Decety, J. (2010). "Feeling" the pain of those who are different from us: modulation of EEG in the mu/alpha range. Cogn. Affect. Behav. Neurosci. 10, 493-504.

Pfurtscheller, G., and Lopes da Silva, F. H. (1999). Event-related EEG/MEG synchronization and desynchronization: basic principles. Clin. Neurophysiol. 110, 1842-1857.

Pfurtscheller, G., Stancak, A. Jr., and Neuper, C. (1996). Event-related synchronization (ERS) in the alpha band - an electrophysiological correlate of cortical idling: a review. Int. J. Psychophysiol. 24, 39-46.

Pineda, J. A. (2005). The functional significance of mu rhythms: translating "seeing" and "hearing" into "doing". Brain Res. Brain Res. Rev. 50, 57-68.

Preston, S. D., and de Waal, F. B. (2002). Empathy: its ultimate and proximate bases. Behav. Brain Sci. 25, 1-20; discussion 20-71.

Rainville, P. (2002). Brain mechanisms of pain affect and pain modulation. Curr. Opin. Neurobiol. 12, 195-204.

Rihs, T. A., Michel, C. M., and Thut, G. (2007). Mechanisms of selective inhibition in visual spatial attention are indexed by alpha-band EEG synchronization. Eur. J. Neurosci. 25, 603-610.

Rossi, S., Tecchio, F., Pasqualetti, P., Ulivelli, M., Pizzella, V., Romani, G. L., Passero, S., Battistini, N., and Rossini, P. M. (2002). Somatosensory processing during movement observation in humans. Clin. Neurophysiol. 113, 16-24.

Saarela, M. V., Hlushchuk, Y., Williams, A. C., Schurmann, M., Kalso, E., and Hari, R. (2007). The compassionate brain: humans detect intensity of pain from another's face. Cereb. Cortex 17, 230-237.

Saitoh, Y., Shibata, M., Sanada, Y., and Mashimo, T. (1999). Motor cortex stimulation for phantom limb pain. Lancet 353, 212.

Salmelin, R., Hamalainen, M., Kajola, M., and Hari, R. (1995). Functional segregation of movement-related rhythmic activity in the human brain. Neuroimage 2, 237-243.

Salmelin, R., and Hari, R. (1994). Spatiotemporal characteristics of sensorimotor neuromagnetic rhythms related to thumb movement. Neuroscience 60, 537-550.

Schack, B., and Klimesch, W. (2002). Frequency characteristics of evoked and oscillatory electroencephalic activity in a human memory scanning task. Neurosci. Lett. 331, 107-110.

Singer, T. (2006). The neuronal basis and ontogeny of empathy and mind reading: review of literature and implications for future research. Neurosci. Biobehav. Rev. 30, 855-863.
Singer, T., Seymour, B., O'Doherty, J., Kaube, H., Dolan, R. J., and Frith, C. D. (2004). Empathy for pain involves the affective but not sensory components of pain. Science 303, 1157-1162.

Steriade, M., Gloor, P., Llinas, R. R., Lopes de Silva, F. H., and Mesulam, M. M. (1990). Report of IFCN committee on basic mechanisms. Basic mechanisms of cerebral rhythmic activities. Electroencephalogr. Clin. Neurophysiol. 76, 481-508.

Thut, G., Nietzel, A., Brandt, S. A., and Pascual-Leone, A. (2006). Alpha-band electroencephalographic activity over occipital cortex indexes visuospatial attention bias and predicts visual target detection. J. Neurosci. 26, 9494-9502.

Ulloa, E. R., and Pineda, J. A. (2007). Recognition of point-light biological motion: mu rhythms and mirror neuron activity. Behav. Brain Res. 183, 188-194.

van Elk, M., Van Schie, H. T., Hunnius, S., Vesper, C., and Bekkering, H. (2008). You'll never crawl alone: neurophysiological evidence for experience-dependent motor resonance in infancy. Neuroimage 43, 808-814.

van Gerven, M., and Jensen, O. (2009). Attention modulations of posterior alpha as a control signal for two-dimensional brain-computer interfaces. J. Neurosci. Methods 179, 78-84.

Wager, T. D., Rilling, J. K., Smith, E. E., Sokolik, A., Casey, K. L., Davidson, R. J., Kosslyn, S. M., Rose, R. M., and Cohen, J. D. (2004). Placebo-induced changes in FMRI in the anticipation and experience of pain. Science 303, 1162-1167.

Worden, M. S., Foxe, J. J., Wang, N., and Simpson, G. V. (2000). Anticipatory biasing of visuospatial attention indexed by retinotopically specific alpha-band electroencephalography increases over occipital cortex. $J$. Neurosci. 20, RC63.

Yang, C.Y., Decety, J., Lee, S., Chen, C., and Cheng, Y. (2009). Gender differences in the mu rhythm during empathy for pain: an electroencephalographic study. Brain Res. 1251, 176-184.

Conflict of Interest Statement: The authors declare that the research was conducted in the absence of any commercial or financial relationships that could be construed as a potential conflict of interest.

Received: 29 March 2011; accepted: 11 August 2011; published online: 04 October 2011.

Citation: Whitmarsh S, Nieuwenhuis ILC, Barendregt HP and Jensen O (2011) Sensorimotor alpha activity is modulated in response to the observation of pain in others. Front. Hum. Neurosci. 5:91 doi: 10.3389/ fnhum.2011.00091

Copyright (C) 2011 Whitmarsh, Nieuwenhuis, Barendregt and Jensen. This is an open-access article subject to a nonexclusive license between the authors and Frontiers Media SA, which permits use, distribution and reproduction in other forums, provided the original authors and source are credited and other Frontiers conditions are complied with. 